\title{
The Role of Youtube as an Information Source About Coronary Artery Disease During COVID-19 Pandemic
}

\author{
๑ Emrah Sevgili, ๑ Corc Baytaroglu \\ Avcilar Hospital, Clinic of Cardiology, Istanbul, Turkey
}

\section{Abstract}

\begin{abstract}
Aim: There are videos on Coronavirus disease-2019 (COVID-19) and coronary artery disease at various quality levels on Youtube. To investigate the quality of Turkish language videos on Youtube about COVID-19 and coronary artery disease.

Methods: The study was conducted between $1^{\text {st }}$ and $3^{\text {rd }}$ September 2020. Two doctors investigated keywords including coronary artery disease, COVID-19", "coronavirus, heart disease", "coronavirus, chest pain", "COVID-19, heartache" and "COVID-19, heart disease". "Coronary artery disease", "heartache", "chest pain" and "heart disease" are the Turkish translations for coronary artery disease, heart pain, chest pain and heart disease, respectively. Firstly, for each video, video length, number of days on Youtube, and the number of views and comments were recorded, along with the number of "likes" and "dislikes". Sources of the videos were categorized into three groups: "health care professionals", "new agencies" and "non-professional individuals". Moreover, DISCERN and medical information and content index $(\mathrm{MICl})$ were evaluated.

Results: Finally, 92 Youtube videos met the study inclusion criteria. The present study included 36 informative videos, 34 patient experience videos and 22 news update videos, and none were categorized in the misleading group. The shortest video length was found in patient experience videos ( $p=0.001$ ). The DISCERN scores of videos were $3.5 \pm 1.1$ for informative videos, $1.8 \pm 0.4$ for patient experience videos and $0.9 \pm 1.3$ for news update videos. The statistical analyses revealed that informative videos had significantly higher DISCERN scores when compared to patient experience videos and news update videos ( $p=0.005$ and $p=0.001$, respectively). The mean $\mathrm{MICl}$ score was $4.1 \pm 1.5$ for informative videos.
\end{abstract}

Conclusion: The present study showed that videos about coronary artery disease and COVID-19 are generally poor quality and low reliability.

Keywords: Coronary artery disease, coronavirus, COVID-19, discern score, MICI, Youtube

\section{Introduction}

The coronavirus infection, which mainly affects the respiratory tract, has become pandemic, infecting almost 60 million between December 2019 and October 2020. (1). The World Health Organization declared coronavirus infection as a Public Health Emergency of International Concern, and many governments passed laws to prevent its spread, including international border closures, public transportation restrictions and the reassigning of general hospitals as specialist pandemic hospitals (2). Postponed outpatient appointments and difficulties in reaching professional health units led patients to seek medical information from other sources including newspapers, television and social media (3).
Online sources, including websites, e-libraries and social media are increasingly used as information tools in today's world. Freeman and Chapman (4) argued that video platforms are preferred information sources overwritten or audio texts. Youtube, a social media application established in 2005, now has video uploads in the billions (5). Previous studies revealed the importance of Youtube videos as information sources about the diagnosis, treatment and follow-up of various diseases. Kumar et al. (6) conducted a cross-sectional study of the content and accuracy of Youtube videos on hypertension, finding a significantly higher view rate for misleading videos. In another study, Bora et al. (7) revealed the poor quality of the information in Youtube videos about the Zika virus pandemic.

Address for Correspondence: Emrah Sevgili, Avcilar Hospital, Clinic of Cardiology, Istanbul,

Turkey

E-mail: emrahsevgilicardio@gmail.com ORCID: orcid.org/0000-0003-4921-2832

Received: 18.03.2021 Accepted: 11.04.2021

'Copyright 2021 by The Medical Bulletin of istanbul Haseki Training and Research Hospital The Medical Bulletin of Haseki published by Galenos Yayınevi. 
Youtube management has no policy of conducting preliminary research on the quality of videos posted on the channel, thus videos can be categorized as containing information that is useful, inadequate or misleading. In the present study, we aimed to investigate the quality of Turkish language videos on Youtube about Coronavirus disease-2019 (COVID-19) and coronary artery disease.

\section{Methods}

\section{Data Collection}

The present study was approved by the Bezmialem University Ethics Committee (date: 10.04.2020, approval number: 2020-105). As no patient data were used in the present study, patient consent was not required. The study was conducted between $1^{\text {st }}$ and $3^{\text {rd }}$ September 2020. Two doctors (ES and CB) investigated keywords including "coronary artery disease, COVID-19", "coronavirus, heart disease", "coronavirus, chest pain", "COVID-19, heartache" and "COVID-19, heart disease". "Coronary artery disease", "heartache", "chest pain" and "heart disease" are the Turkish translations for coronary artery disease, heart pain, chest pain and heart disease, respectively. The data consisted of videos of between 2 and 15 minutes long. The video ranking system reveals that popularity increases for videos of at least 2 minutes, reaching their highest level at 15-16 minutes. Totally, 149 videos were found that met the length criteria. Videos with any language other than Turkish, and videos with irrelevant content were excluded from the study, leaving a total of 92 videos, which were recorded on a specific playlist, and carefully analyzed independently by two cardiologists.

\section{Data Analysis}

Firstly, for each video, video length, number of days on Youtube, and the number of views and comments were recorded, along with the number of "likes" and "dislikes". Sources of the videos were categorized into three groups: "health care professionals", "new agencies" and "nonprofessional individuals". Moreover, target groups were categorized as either "patients" or "healthcare workers". Four classifications were determined according to content, as follows. Videos which included accurate information about epidemiology, pathophysiology, symptoms, prevention methods and proven treatment alternatives were considered as an informative group. Videos with patients" stories of coronavirus were classified as personal experience group, and video news uploaded by news channels, as news update group. Finally, videos with misleading information were defined as personal propaganda.

Previous reports have used the DISCERN scores (from 0 point to 5 point) to achieve objective analysis about video quality, utility and reasonableness of information. The model included five yes/no questions. Each "yes" answer demonstrates a positive perspective, and counted as one point, "no" answers scored zero. Additionally, medical information and content index (MICl) was used to analyze the video content. The survey was scored for each video from 1 to 5, according to the content on disease prevalence, transmission information, clinical symptoms, screening and/or testing, and treatment results. Both authors used the survey mentioned above to determine the type, efficiency and quality of videos.

\section{Statistical Analysis}

Statistical analysis was done with the Statistical Package for the Social Sciences version 25.0 (SPSS IBM Corp., Armonk, NY, USA). Normality of distribution of the Variables was evaluated by Shapiro-Wilk test and Q-Q plots. One-way ANOVA test was preferred for comparison of the normally distributed variables, and non-normally distributed values were evaluated with Kruskal-Wallis test. Quantitative data are expressed as mean \pm standard deviation values. Categorical variables were classified and analyzed using the $\chi^{2}$ test or Fisher's Exact test. Post hoc analysis was done using the Games-Howell test. The Fleiss and weighted kappa (к) were used to evaluate the inter-observer concurrence. The data were analyzed at $95 \%$ confidence level and p-value of less than 0.05 was accepted as statistically significant.

\section{Results}

The final analysis revealed that 92 Youtube videos met the study inclusion criteria. A total of 57 videos were excluded for various reasons: 7 videos were in languages other than Turkish, 8 had irrelevant content, and 42 had inadequate duration. The present study included 36 informative videos, 34 patient experience videos and 22 news update videos, and none were categorized in the misleading group (Figure 1).

Informative videos were most frequently watched, but difference was not statically significant $(p=0.558)$. Similarly, duration of videos on Youtube, number of likes and dislikes, and number of comments were comparable between groups $(p=0.244, p=0.804, p=0.953$ and $p=0.678$, respectively). The shortest video length was found in patient experience videos $(p=0.001)$. For the informative and news update categories, most were uploaded by news agencies, while only $22 \%$ of informative videos were uploaded professional health care individuals. Additionally, most patient experience videos were uploaded by nonprofessionals $(p=0.286)$. The great majority of videos targeted patients: $88.9 \%$ of informative videos, $91.2 \%$ of patients experience videos, and $90.0 \%$ of news update videos $(\mathrm{p}=0.942)$ (Table 1). 
Videos viewed in full for eligibility assessment $(n=149)$

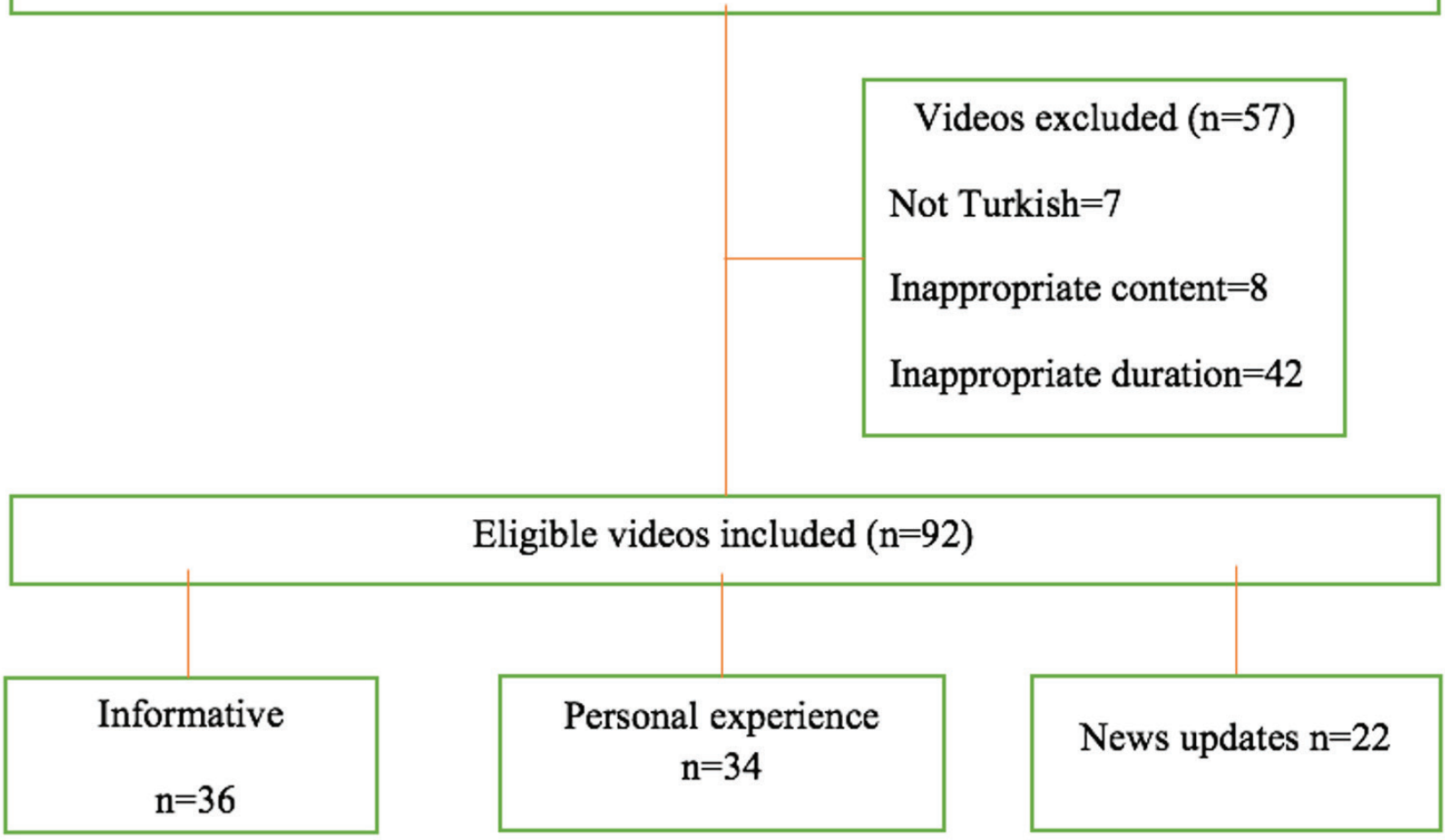

Figure 1. Flowchart of the study

\begin{tabular}{|c|c|c|c|c|c|}
\hline Characteristics & Total & Informative & $\begin{array}{l}\text { Patient } \\
\text { experience }\end{array}$ & News update & p \\
\hline Number of videos & 92 & 36 & 34 & 22 & \\
\hline \multicolumn{6}{|l|}{ Audience interaction parameters } \\
\hline Number of views, median (IQR) & $201(100-548)$ & $176(102-480)$ & $243(120-452)$ & $215(55-693)$ & 0.558 \\
\hline Video length (minimum), median (IQR) & $3.1(2.3-4.3)$ & $3.4(2.7-4.9)$ & $2.4(2.1-3.1)$ & $3.9(2.4-5.0)$ & $0.001^{\mathrm{a}}$ \\
\hline Duration on Youtube (days), median (IQR) & $135(125-140)$ & $136(97-174)$ & $135.5(100-152)$ & $131(98-146)$ & 0.244 \\
\hline Likes, median (IQR) & $45(21-118)$ & $47(19-107)$ & $51(20-187)$ & $37(25-71)$ & 0.804 \\
\hline Dislikes, median (IQR) & $0.5(0-6)$ & $0(0-6)$ & $1.5(0-7)$ & $0(0-2)$ & 0.953 \\
\hline Comments, median (IQR) & $0(0-2)$ & $0(0-4)$ & $0(0-4)$ & $0(0-1)$ & 0.678 \\
\hline DISCERN score, mean \pm standard deviation & $2.6 \pm 1.5$ & $3.5 \pm 1.1$ & $1.8 \pm 0.4$ & $0.9 \pm 1.3$ & $0.001^{b}$ \\
\hline Source of upload & - & - & - & - & 0.286 \\
\hline Professional individuals & $20(21.7 \%)$ & $8(22.2 \%)$ & $9(26.5 \%)$ & $3(13.6 \%)$ & - \\
\hline Non-professional individuals & $33(35.9 \%)$ & $12(33.3 \%)$ & $15(44.1 \%)$ & $6(27.2 \%)$ & - \\
\hline News agencies & $39(42.4 \%)$ & $16(44.4 \%)$ & $10(29.4 \%)$ & $13(59.1 \%)$ & - \\
\hline Target audience & - & - & - & - & 0.942 \\
\hline For doctors and healthcare providers & $9(9.8 \%)$ & $4(11.1 \%)$ & $3(8.8 \%)$ & $2(9.1 \%)$ & - \\
\hline For patients & $83(90.2 \%)$ & $32(88.9 \%)$ & $31(91.2 \%)$ & $20(90.9 \%)$ & - \\
\hline \multicolumn{6}{|c|}{$\begin{array}{l}\text { a: Video length was significantly shorter in the patient experience video group with One-Way ANOVA test. } \\
\text { b:DISCERN score was significantly higher in the informative video group with One-Way ANOVA test. } \\
\text { IQR: Interquartile range }\end{array}$} \\
\hline
\end{tabular}


The DISCERN scores of videos were $3.5 \pm 1.1$ for informative videos, $1.8 \pm 0.4$ for patient experience videos and $0.9 \pm 1.3$ for news update videos. The statistical analyses revealed that informative videos had significantly higher DISCERN scores when compared to patient experience videos and news update videos ( $p=0.005$ and $p=0.001$, respectively) (Table 2 ). Clinical symptoms and disease transmission information were the most frequently discussed content in informative videos $(73.9 \%$ and $85.9 \%$, respectively). Additionally, 32 videos and 15 videos, respectively, gave information about the prevalence of COVID-19, and about screening tests. The mean $\mathrm{MICl}$ score was $4.1 \pm 1.5$ for informative videos (Table 3). The kappa coefficient of agreement regarding for DISCERN score was $0.81 \quad(p<0.001)$ and for $\mathrm{MICl}$ score was 0.82 $(p<0.001)$ (Table 4).

\section{Discussion}

Widespread internet usage has radically changed our daily habits regarding shopping, professional activities, and accessing information about disease (8). According to current Youtube statistics, 19 of 20 internet users watch

\begin{tabular}{|c|c|c|c|}
\hline \multirow[b]{2}{*}{ Characteristics } & \multicolumn{3}{|l|}{$p$} \\
\hline & $\begin{array}{l}\text { Informative } \\
\text { vs Patient } \\
\text { experience }\end{array}$ & $\begin{array}{l}\text { Informative vs } \\
\text { News update }\end{array}$ & $\begin{array}{l}\text { Patient } \\
\text { experience vs } \\
\text { News update }\end{array}$ \\
\hline Video lenght & $0.001^{a}$ & 0.986 & $0.001^{a}$ \\
\hline DISCERN score & $0.005^{a}$ & $0.001^{a}$ & 0.268 \\
\hline \multicolumn{4}{|c|}{$\begin{array}{l}\text { a. Post-hoc analysis (Games-Howell test), Values of } p<0.05 \text { was accepted as } \\
\text { statistically significant and marked bold }\end{array}$} \\
\hline
\end{tabular}

\begin{tabular}{|c|c|c|}
\hline Component of $\mathrm{MICl}$ scale & $\begin{array}{l}\text { Videos with } \\
\text { information }\end{array}$ & MICl score* \\
\hline Prevalence & $32(34.8)$ & $0.9 \pm 0.5$ \\
\hline Transmission & 79 (85.9) & $1.2 \pm 0.6$ \\
\hline Clinical symptoms & $68(73.9)$ & $1.0 \pm 0.6$ \\
\hline Screening/tests & $15(16.3)$ & $0.4 \pm 0.5$ \\
\hline Treatment/outcomes & $54(58.7)$ & $0.9 \pm 0.7$ \\
\hline \multicolumn{2}{|l|}{ Total MICl score } & $4.1 \pm 1.5$ \\
\hline \multicolumn{3}{|c|}{ *:Mean \pm standard deviation, $\mathrm{MICl}$ : Medical information and content index } \\
\hline
\end{tabular}

Table 4. The inter-observer agreement for DISCERN and MICI score

\begin{tabular}{|l|l|l|l|}
\hline & $\kappa$ coefficients & $\mathbf{9 5 \%} \mathbf{C I}$ & $\mathbf{p}$ \\
\hline DISCERN score & $0.81^{\mathrm{a}}$ & $0.76-0.85$ & $\mathbf{0 . 0 0 1 ^ { \mathrm { a } }}$ \\
\hline $\mathrm{MICI}$ score & $0.82^{\mathrm{a}}$ & $0.77-0.87$ & $\mathbf{0 . 0 0 1}^{\mathrm{a}}$ \\
\hline
\end{tabular}

a: There is very high agreement among observers, $\mathrm{MICl}$ : Medical information and content index, $\mathrm{Cl}$ : Confidence interval
Youtube videos (9). The COVID-19 pandemic highlighted the need to investigate the accuracy of Youtube videos about COVID-19 and cardiac disease, because the introduction of travel restrictions and difficulties in reaching the professional health care system meant that people were more likely to access such videos.

Previous reports proved and externally validated the usage of DISCERN score in the evaluation of video quality as an information source. The DISCERN score takes values between 1 and 5 . Higher values indicate better quality of information content. Ferhatoglu et al. (10) investigated Youtube videos on sleeve gastrectomy, finding that content uploaded by professional health workers had significantly higher DISCERN scores. Similarly, for Youtube videos on pregnancy and COVID-19, Yuksel and Cakmak (11) found significantly better DISCERN scores for videos which were produced by health care providers. In accordance with aforementioned studies, we found significantly higher DISCERN score in informative videos in comparison with patient experience videos and news update videos ( $p=0.005$ and $p=0.001$, respectively).

The $\mathrm{MICl}$ was first described by Nagpal et al. (12) to assess the video content quality during Ebola pandemic. The chart indicates that scores are awarded 1-5 points for each of five components: prevalence, transmission, clinical symptoms, screening/testing, and treatment outcomes of the disease. Although there is no cut-off value in the $\mathrm{MICl}$ score, higher values are associated with better content. Atac et al. (13) evaluated $\mathrm{MICl}$ scores of videos about the COVID-19 pandemic and found a 3.33 score for videos in Turkish and 2.76 for videos in English. In another study by Dutta et al. (14) a mean $\mathrm{MICl}$ score was 5.68 was found for videos in six different languages: Arabic, Bengali, Dutch, English, Hindi and Nigerian. Wide variations were found for $\mathrm{MICl}$ scores for videos about the COVID-19 pandemic in different studies, and we suggest that this is due to content quality improving over time. The present study is the first to evaluate the quality of Youtube videos about coronary artery disease and COVID-19, and the result was a $\mathrm{MICl}$ score of 4.1 .

Previous studies about information sources in Youtube videos present conflicting outcomes. According to Atac's study, of all the useful medical videos on Youtube, most (76.1\%) were shared by news channels, and only $8.7 \%$ by professional health care providers (13). In contrast, in Yuksel and Cakmak (11) study, 73.3\% of informative videos were produced by physicians, and only $20 \%$ by new agencies. In our study, we did not find significantly different according to the source of video upload, and most of the informative videos were uploaded by new agencies. However, we believe that the increasing numbers 
of Youtube videos by professional healthcare professionals will eventually improve the overall quality of the videos.

\section{Study Limitations}

The present study is novel in that it is the first to assess Youtube videos about coronary artery disease and COVID-19; nevertheless, there are some limitations. Firstly, we evaluated videos only in Turkish, without a comparison with videos in other languages. Additionally, after six months of the COVID-19 pandemic in Turkey, new videos containing the most recent information are being uploaded. The quality of videos produced during different stages of the COVID-19 pandemic may be the subject of another study. Lastly, we chose five keywords, however, the inclusions of other terms related to cardiac disease and COVID-19 would provide a greater range of videos.

\section{Conclusion}

Youtube videos are accessible and can be a valuable information source on coronary artery disease and COVID-19 for patients and their relatives. However, the present study showed that videos about coronary artery disease and COVID-19 are generally poor quality and low reliability. We believe that with greater efforts towards standardization and improvement, Youtube videos could become regarded as valuable information tools on COVID-19 possible effects on coronary artery disease.

\section{Authorship Contributions}

Concept: E.S., Design: E.S., Data Collection or Processing: C.B., Analysis or Interpretation: E.S., Literature Search: C.B., Writing: E.S.

Conflict of Interest: No conflict of interest was declared by the authors.

Financial Disclosure: The authors declared that this study received no financial support.

\section{References}

1. Demir M, Taken K, Eryilmaz R, Aslan R, Ertas K. YouTube as a Health Information Source: COVID-19 and Andrology. Med Bull Haseki 2021;59:91-5.

2. Yuksel B, Ozgor F. Effect of the COVID-19 pandemic on female sexual behavior. Int J Gynaecol Obstet 2020;150:98-102.
3. Tsao SF, Chen $H$, Tisseverasinghe $T$, Yang $Y$, Li L, Butt ZA. What social media told us in the time of COVID-19: a scoping review. Lancet Digit Health 2021;3:175-94.

4. Freeman B, Chapman S. Is "YouTube" telling or selling you something? Tobacco content on the YouTube video-sharing website. Tob Control 2007;16:207-10.

5. ebizmba.com. Available from: http://www.ebizmba.com/ articles/most-popular-websites

6. Kumar N, Pandey A, Venkatraman A, Garg N. Are video sharing web sites a useful source of information on hypertension? J Am Soc Hypertens 2014;8:481-90.

7. Bora K, Das D, Barman B, Borah P. Are internet videos useful sources of information during global public health emergencies? A case study of YouTube videos during the 2015-16 Zika virus pandemic. Pathog Glob Health 2018;112:320-8.

8. Yuce MÖ, Adalı E, Kanmaz B. An analysis of YouTube videos as educational resources for dental practitioners to prevent the spread of COVID-19. Ir J Med Sci 2021;190:19-26.

9. Statistics YouTube. Available from: https://www. omnicoreagency.com/youtube-statistics/.

10. Ferhatoglu MF, Kartal A, Ekici U, Gurkan A. Evaluation of the Reliability, Utility, and Quality of the Information in Sleeve Gastrectomy Videos Shared on Open Access Video Sharing Platform YouTube. Obes Surg 2019;29:1477-84.

11. Yuksel B, Cakmak K. Healthcare information on YouTube: Pregnancy and COVID-19. Int J Gynaecol Obstet 2020;150:189-93.

12. Nagpal SJ, Karimianpour A, Mukhija D, Mohan D, Brateanu A. YouTube videos as a source of medical information during the Ebola hemorrhagic fever epidemic. Springerplus 2015;4:457.

13. Atac O, Ozalp YC, Kurnaz R, Güler OM, Inamlık M, Hayran O. Youtube as an Information Source During the Coronavirus Disease (COVID-19) Pandemic: Evaluation of the Turkish and English Content. Cureus 2020;12:e10795.

14. Dutta A, Beriwal N, Van Breugel LM, et al. YouTube as a Source of Medical and Epidemiological Information During COVID-19 Pandemic: A Cross-Sectional Study of Content Across Six Languages Around the Globe. Cureus 2020;12:e8622. 